\title{
ANÁlisis POLÍNICO DE MIELES INMADURAS EN EL SECTOR OESTE DE LAS YUNGAS DE JUJUY (ARGENTINA)
}

\author{
MAGALI V. MENDEZ¹ ${ }^{1}$ ANA C. SANCHEZ1, FABIO F. FLORES ${ }^{2}$ y LILIANA C. LUPO ${ }^{1,2}$
}

\begin{abstract}
Summary: Pollen analysis of immature honeys in the Western Sector of the Yungas in Jujuy (Argentina). With the aim of knowing the nectar resources used by Apis mellifera $\mathrm{L}$. in the western of the Yungas in Jujuy, 14 samples of immature honey and two of mature honey of periods 2011-2012 and 2012-2013, pertaining to a apiary of Tilquiza, were examined. That samples were processed according to conventional melissopalynologcal techniques with subsequent acetolysis. A total of 53 pollen types belonging to 31 botanical families were identified being Asteraceae and Fabaceae the most well represented. As dominant source were identified Crinodendron tucumanum in september 2011, Blepharocalyx salicifolius in november and december 2011 and type Scutia/Condalia in october 2012. As second contributions were identified Allophylus edulis, Zanthoxylum, Parapiptadenia excelsa, Baccharis, Gnaphalium, Eucalyptus and type Cissus, highlighting the influence of the native flora of the Yungas. From this study it was known to understand the dynamics of income nectar to the hive and evaluate those resources that are not present in mature honey and which are important for the development of the hive.
\end{abstract}

Key words: Nectar, mellisopalynology, Apis mellifera, Yungas

Resumen: Con el objetivo de conocer los recursos nectaríferos utilizados por Apis mellifera L. en el sector oeste de las Yungas, se examinaron 14 muestras de miel inmadura y dos de miel madura correspondientes a los períodos apícolas 2011-2012 y 2012-2013, pertenecientes a un apiario de la localidad de Tilquiza (Jujuy). Las muestras fueron procesadas de acuerdo a las técnicas melisopalinológicas convencionales con posterior acetólisis. Se identificaron un total de 53 tipos polínicos pertenecientes a 31 familias botánicas, siendo las familias con mayor riqueza polínica Asteraceae y Fabaceae. Se identificaron como recursos dominantes a Crinodendron tucumanum en setiembre de 2011, Blepharocalyx salicifolius en noviembre y diciembre de 2011 y al tipo Scutia/Condalia en octubre de 2012. Como aportes secundarios se registraron a Allophylus edulis, Zanthoxylum, Parapiptadenia excelsa, Baccharis, Gnaphalium, Eucalyptus y tipo Cissus, destacándose la influencia de la flora nativa de las Yungas. A partir de este estudio se pudo conocer la dinámica del ingreso de néctar a la colmena y valorar aquellos recursos que no se encuentran presentes en las mieles maduras y que son de importancia para el desarrollo de la colmena.

Palabras clave: Néctar, melisopalinologia, Apis mellifera, Yungas.

\section{INTRODUCCION}

El conocimiento de la flora utilizada por las abejas en un área determinada es fundamental para planificar el aprovechamiento de la misma de un

\footnotetext{
${ }^{1}$ Instituto de Ecoregiones Andinas (INECOA), Universidad Nacional de Jujuy - CONICET, Facultad de Ciencias Agrarias, (Alberdi $47 \mathrm{~B}^{\circ}$ Los Naranjos, 4.600), S. S. de Jujuy, Argentina. E mail magalivmendez@gmail.com, lab.palinologia@fca.unju.edu.ar.

2 Catedra de Ecología General, Facultad de Ciencias Agrarias, UNJu. Alberdi 47 C. P. 4600. S. S. de Jujuy, Jujuy, Argentina.
}

modo racional, contribuyendo a un buen manejo del colmenar y potenciar la obtención de mieles de diferentes orígenes botánicos. Así áreas con importante disponibilidad y diversidad de recursos como los bosques tropicales y subtropicales son de gran importancia en cuanto a la oferta de néctar y polen a partir de sus floraciones, siendo relevante para la producción de miel (Chamorro García et al., 2013).Por otra parte, los estudios melisopalinológicos contribuyen al conocimiento de las especies melíferas, brindando valor agregado a la miel a través de la determinación del origen botánico y geográfico de la misma. 
En la Provincia de Jujuy, Apis mellifera L. se encuentra adaptada a los distintos ambientes practicándose la apicultura comercial en sectores de Yungas, Chaco y Prepuna. Si bien la producción de miel es menor en comparación a otras regiones del país (MAGYP, 2015), los apicultores tienen la posibilidad de producir mieles diferenciadas que reflejan la vegetación única y característica de las distintas áreas. A través de diferentes proyectos de investigación, llevados a cabo en la provincia de Jujuy, se determinaron zonas de valor apícola a partir del estudio del contenido polínico de muestras comerciales (Sánchez, 2013), constituyendo el primer aporte melisopalinológico para la provincia. Una de las zonas de importancia corresponde a la provincia fitogeográfica de las Yungas (denominada zona II), que geográficamente se extiende al pie de las laderas bajas al Este de la provincia, y la asociación polínica indicadora corresponde a Myrtaceae (de origen nativo), Parapiptadenia excelsa, Baccharis, Salix humboldtiana, Allophylus edulis, Scutia/Condalia y Zanthoxylum (Sánchez, 2013).

En cuanto a los estudios del contenido polínico en mieles inmaduras a lo largo de un periodo productivo, en nuestro país solo se registran antecedentes para el Delta del Paraná (Basilio \&Romero, 2002), el valle inferior del río Chubut en la Patagonia (Forcone et al., 2003), la región del Monte en la Provincia de la Pampa (Naab \& Tamame, 2007), la provincia fitogeográfica Chaqueña (Salgado et al., 2014) y en la provincia de Jujuy para la zona de Chaco Serrano (Burgos \& Sánchez, 2014).La importancia de este tipo de análisis reside en detectar las plantas utilizadas tempranamente por las abejas y que por lo general no están representadas en la miel madura cosechada, debido a que su néctar es consumido durante el periodo de crecimiento de la colmena (Salgado et al., 2014), para valorizarlas como recurso apícola. A partir de estos antecedentes y con el objetivo de determinar el flujo de néctar que ingresa en un colmenar y su uso a lo largo del periodo productivo, se presentan los resultados obtenidos de los análisis palinológicos de mieles inmaduras provenientes del sector oeste de las Yungas en Jujuy.

\section{Materiales y Métodos}

Área de estudio

El estudio se realizó en un apiario ubicado en la localidad de Tilquiza $\left(24^{\circ} 05^{\prime} \mathrm{S}\right.$ y $65^{\circ} 17^{\prime} \mathrm{O}, 1500$ msnm) a $17 \mathrm{~km}$ al noroeste de la ciudad de San Salvador de Jujuy por la ruta provincial $\mathrm{N}^{\circ} 35$, en el departamento Dr. Manuel Belgrano.

Fisiográficamente, es un área montañosa con un relieve fuertemente colinado y presenta un suelo poco desarrollado, con un $\mathrm{pH}$ neutro a moderadamente alcalino, alto contenido de materia orgánica - por el aporte constante de la vegetación característica del lugar - y una pendiente que oscila entre el 12 y $25 \%$.

El clima es Cálido Subtropical Serrano con temperaturas medias de $25^{\circ} \mathrm{C}$ en diciembre y $13^{\circ}$ $\mathrm{C}$ en junio, con una precipitación media anual de 700 a $1000 \mathrm{~mm}$ concentradas en la época estival (Chafatinos \& Nadir, 1990).

Desde el punto de vista fitogeográfico, la vegetación pertenece al Distrito de Selva Montana de la Provincia Fitogeográfica de las Yungas (Cabrera, 1976); entre sus especies se pueden mencionar a arbóles como Allophylus edulis (A. St.-Hil., A. Juss. \& Cambess.) Hieron. ex Niederl., Blepharocalyx salicifolius (Kunth) O. Berg, Celtis brasiliensis (Gardner) Planch., Ilex argentina Lillo, Juglans autralis Griseb., Prunus tucumanensis Lillo, Zanthoxylum coco Gillies ex Hook. f. \& Arn., entre otros. Entre las especies arbustivas y herbáceas se encuentran Baccharis salicifolia (Ruiz \& Pav.) Pers., Begonia boliviensis A. DC., Boehmeria caudata Sw., Cestrum lorentzianum Griseb., Elephantopus mollis Kunth, Piper tucumanum C. DC, Seemannia gymnostoma (Griseb.) Toursark, Senecio boomani R.E. Fr., Urera baccifera (L.) Gaudich., Vernonia pinguis (Griseb.) H. Rob., entre otros.

Por sus características topográficas y edáficas es un área no apta para la agricultura pero en general admite el desarrollo de la actividad forestal, especialmente de especies de Pinus y Eucalyptus, acompañada de la ganadería de subsistencia (Braun Wilke et al., 2001). En esta área también se realiza la apicultura en forma comercial y San Salvador de Jujuy es el principal mercado para la miel.

\section{Obtención de muestras}

Se obtuvieron 14 muestras de miel inmadura mediante el uso de jeringas esterilizadas de $10 \mathrm{~cm}^{3}$ y colectadas mensualmente en 5 colmenas a lo largo de dos periodos productivos, entre setiembre y marzo de 2011-2012 y 2012-2013. Durante el procedimiento y con el fin de no contaminar 
las muestras con polen, se tuvo la precaución de colectar la miel en celdillas alejadas de aquellas de almacenamiento de polen. Además, con el fin de obtener información sobre la representatividad de los diferentes tipos polínicos encontrados en las mieles inmaduras y su relación con la miel madura , se analizaron dos muestras correspondientes a las cosechas realizadas en cada temporada apícola, obtenidas por centrifugado manual y homogeneizadas.

\section{Laboratorio y microscopía}

El procesamiento de las muestras se realizó según la técnica propuesta por Louveaux et al. (1978) con posterior acetólisis (Erdtman, 1960).

Las observaciones se realizaron con microscopios ópticos Zeiss ISC KS2 y Leica DM 500, con una magnificación de 400X y las microfotografías fueron obtenidas mediante el empleo de una cámara digital Leica ICC50 incorporada al microscopio Leica DM 500.

La identificación de los tipos polínicos se efectuó con el empleo de atlas palinológicos (Markgraf \& D' Antoni, 1978; Heusser, 1971 y Pire et al., 1998; 2002; 2006) y la confrontación con la Palinoteca de Referencia del Laboratorio de Palinología, Facultad de Ciencias Agrarias, UNJu (PAL-JUA). En aquellos casos en los que no fue posible determinar una categoría, se utilizó el término "tipo" para indicar que el polen tiene semejanza con alguna especie, género o familia botánica. La nomenclatura de los tipos polínicos se estableció de acuerdo a Joosten \& De Klerk (2002) y De Klerk \& Joosten (2007). Para establecer la riqueza polínica de cada muestra se adaptó el índice de riqueza específica (S) propuesto por Moreno (2001).

\section{Análisis cualitativo}

En cada muestra de miel se efectuó el conteo de un mínimo de 600 granos de polen (hasta la estabilización del porcentaje) y se establecieron las clases de frecuencia según Louveaux et al. (1978): dominante (D): $>45 \%$, secundario (S): 16 $45 \%$, menor importancia (M): $3-15 \%$ y trazas (T): $<3 \%$. Los tipos polínicos procedentes de plantas anemófilas y sin néctar se excluyeron del recuento para la determinación de las clases de frecuencia de los tipos polínicos de las especies nectaríferas , debido a que no aportan en la caracterización botánica de la miel (Louveaux et al., 1978); sin embargo, su presencia en la miel fue utilizada como indicador de la procedencia geográfica (Barth, 1989).

La frecuencia de ocurrencia de los tipos polínicos en el total de las muestras fue determinada de acuerdo a Feller Demalsy et al. (1987), como muy frecuente (MF: $>50 \%$ de las muestras), frecuente (F: $>20-50 \%)$, poco frecuente (PF: $10-20 \%$ ) o raro $(\mathrm{R}:<10 \%)$.

Para cada cada tipo polínico se estableció el estatus según el Catálogo de Plantas Vasculares del Conosur (Zuloaga et al., 2008).

\section{Análisis cuantitativo}

Para el análisis cuantitativo de las mieles inmaduras, se adaptó la metodología utilizada para mieles maduras de Apis mellifera. Para ello, mediante el agregado de dos tabletas de Lycopodium (Stockmarr, 1971; Moar, 1985) y el conteo de sus esporas y el total de granos de polen presentes en las muestras, se determinó la concentración polínica (número de granos de polen/10 gr de miel). Estos análisis se realizaron con la finalidad de aportar a la comparación de las mieles producidas en los dos periodos de estudio.

\section{Análisis de datos}

Con el fin de indagar sobre la frecuencia, variabilidad y abundancia de los tipos polínicos en las muestras y a lo largo de los periodos productivos, para las mieles inmaduras se elaboró un diagrama polínico y se aplicó un análisis de agrupamiento usando el coeficiente de disimilitud de Edwards \& Cavalli-Sforza. Para ello, utilizando el programa Tilia 1.7.16 (Grimm, 1992), en primera instancia se confeccionó una matriz de datos, ingresando cronológicamente las 14 muestras de miel y 33 variables pertenecientes a los tipos polínicos con porcentajes superiores al 3\%. Se retiraron del análisis aquellos tipos con valores inferiores al $3 \%$, cuya procedencia se considera ocasional en las muestras, provenientes del aire, de residuos polínicos de las celdas de almacenamiento, entre otros. Además se incorporó al diagrama polínico (sin formar parte del agrupamiento) las muestras de mieles maduras cosechadas en ambos periodos estudiados para ilustrar así, las semejanzas y diferencias de las muestras

Utilizando el programa PAST (Hammer et al., 2001) y para cada temporada, se determinó 
la similitud entre el espectro polínico de la miel inmadura obtenida en los meses previos a la muestra cosechada (miel madura), y esta última , efectuando un análisis de agrupamiento con el índice de similitud cuantitativo de Morishita, cuyos valores se expresan de 0 a 1 e indicando muestras disimiles a símiles respectivamente.

Para comparar la concentración de polínica en ambos periodos estudiados se aplicó un test de Student.

\section{Resultados}

Del total de tipos polínicos identificados, el $62 \%$ pertenece a la flora nativa, el $10 \%$ a la exótica y el $28 \%$ a tipos de origen indeterminado. Se identificaron un total de 53 tipos polínicos: 16 a nivel de especie, 23 a nivel de género, 13 a nivel de familia, uno a nivel de clase botánica y se agruparon en la categoría de indeterminados, cuatro tipos polínicos sin identificar. Se registraron 8 tipos polínicos procedentes de plantas con polinización anemófila. En mieles inmaduras la riqueza polínica por muestra varió entre 6 y 17 con un promedio de 13 tipos polínicos, mientras que en mieles maduras fue de 11 para el periodo 2011-2012 y de 17 para el 2012-2013 (Tabla 1). En los tipos polínicos identificados se encuentran representadas 31 familias botánicas siendo las de mayor riqueza Asteraceae con 7 y Fabaceae con 6. Las familias de interés por su dominancia polínica fueron Myrtaceae, Rhamnaceae y Eleocarpaceae.

Como tipos polínicos dominantes se identificaron a Crinodendron tucumanum, registrado en la muestra de Setiembre de 2011, Blepharocalyx salicifolius en noviembre y diciembre del mismo año y tipo Scutia/ Condalia en octubre de 2012. En el caso de las muestras de febrero y marzo de 2013, el tipo polínico Ligustrum se presentó como polen dominante.

De acuerdo a la frecuencia de ocurrencia de los tipos polínicos en las muestras, para el periodo 2011-2012 se clasificaron como muy frecuentes: Allophylus edulis, Blepharocalyx salicifolius, Parapiptadenia excelsa, Salix humboldtiana, Sebastiania, Anadenanthera colubrina, Baccharis, Crinodendron tucumanum y Eucalyptus; los cuatro primeros tipos polínicos se encontraron en el $100 \%$ de las muestras. En el período 2012-2013 los pólenes muy frecuentes fueron S. humboldtiana,
Sebastiania, Baccharis, A.edulis, Eucalyptus y Escallonia schreiteri. Los principales tipos polinico se encuentran representados en la Fig. 3.

\section{Análisis de agrupamiento}

De acuerdo al análisis de agrupamiento efectuado en mieles inmaduras, se determinaron dos grupos principales de muestras: A y B. El grupo A, a su vez se dividio en dos sub grupos (A1 y A2). En el caso del grupo B, que agrupo las muestras de febrero y marzo de 2013, se caracterizó por la presencia dominante de Ligustrum no presente en los restantes grupos al igual que Clematis. La ausencia de tipos polínicos como Blepharocalyx salicifolius, Allophylus edulis y Pararpiptadenia excelsa, Crinodendron tucumanum, entre otros, también contribuyen a caracterizar este grupo. Todos ellos por su abundancia y ocurrencia son los que definen al grupo A. El subgrupo A1 que corresponde a las muestras del periodo productivo 2011-2012, a diferencia del A2 (setiembre2012 a enero 2013), presenta los siguientes tipos polínicos Parapiptadenia excelsa (Muy frecuente a lo largo de la temporada), Mimosa, Zanthoxylum y Myrtaceae. Respecto al subgrupo A2, se encuentran caracterizándolo tipos polínicos como tipo Scutia Condalia, Escallonia schreiteri, tipo Cissus, Vassobia breviflora, Gnaphalium y Cactaceae. (Fig. 1)

\section{Análisis de similitud}

El análisis de similitud entre las muestras de miel inmadura y la muestra de miel madura en el periodo 2011-2012 mostró una alta similitud $(0,96)$ entre el espectro polínico de ésta última y el correspondiente al mes anterior a la cosecha (noviembre). En relación a los meses de setiembre y octubre, la similitud es menor, estando en ellas representados tipos polínicos que no se encuentran en la miel madura. El mismo patrón se refleja para la temporada 2012-2013 en el que la muestra comercial (correspondiente al mes de noviembre) presenta una alta similitud $(0,72)$ respecto de la miel inmadura del mes anterior (octubre) (Fig. 2).

Los tipos polínicos que no se encuentran representados en la miel madura, pero si forman parte del ingreso de néctar en los meses anteriores para la temporada 2011-2012 son los siguientes: Acanthaceae, Xilosma pubescens, Schinus, Senecio, Sapium haematospermum y Mimosa farinosa (en valores trazas); Baccharis Brassicaceae, 
Tabla 1. Tipos polínico identificados en las mieles inmaduras (MI) y maduras (MM) de los periodos productivos 2011-2012 y 2012-2013 en el Apiario Barro Blanco de la localidad de Tilquiza (Jujuy).

Clases de frecuencias D: polen dominante ( $>45 \%)$; S: polen secundario (15-45\%); M: polen de menor importancia $(13-15 \%)$; T: polen traza $(<3 \%)$. FO: frecuencias de ocurrencia en el total de muestras analizadas. ${ }^{*}$ Tipos polínicos de origen nativo. + Presencia en la muestra de polen de plantas con polinización anemófila o sin néctar.

\begin{tabular}{|c|c|c|c|c|c|c|c|c|c|c|c|c|c|c|c|c|c|c|}
\hline \multirow{3}{*}{ TIPOS POLINICOS } & \multicolumn{9}{|c|}{ 2011-2012 } & \multicolumn{9}{|c|}{$2012-2013$} \\
\hline & \multicolumn{8}{|c|}{ MI } & \multirow{2}{*}{$\begin{array}{c}\text { MM } \\
\text { D }\end{array}$} & \multicolumn{8}{|c|}{ Ml } & \multirow{2}{*}{$\begin{array}{c}\mathrm{MM} \\
\mathrm{N}\end{array}$} \\
\hline & $S$ & 0 & $\mathrm{~N}$ & $D$ & $E$ & $\mathrm{~F}$ & M & $\mathrm{FO} \%$ & & S & 0 & $\mathrm{~N}$ & $D$ & $E$ & $\mathrm{~F}$ & M & $\mathrm{FO} \%$ & \\
\hline Acanthaceae & & $\mathrm{T}$ & & & & & & 14 & & & & & & & & & & \\
\hline \multicolumn{19}{|l|}{ Anacardiaceae } \\
\hline Schinus * & & & $\mathrm{T}$ & $\mathrm{T}$ & & & & 29 & & & & & & & & & & $\mathrm{~T}$ \\
\hline Asteraceae & & $\mathrm{T}$ & & & M & $\mathrm{T}$ & & 43 & M & T & & $\mathrm{T}$ & & & & M & 43 & $\mathrm{~T}$ \\
\hline Senecio * & & & $\mathrm{T}$ & $\mathrm{T}$ & & & & 14 & & $\mathrm{~T}$ & $\mathrm{~T}$ & $\mathrm{~T}$ & & & & & 43 & \\
\hline Baccharis * & M & & $\mathrm{T}$ & $\mathrm{T}$ & & & M & 57 & & T & $\mathrm{T}$ & M & & $S$ & M & & 71 & M \\
\hline Viguiera * & & & & & & & $\mathrm{T}$ & 14 & & & & & & & & & & \\
\hline Eupatorium * & & & & & & & & & & & & & M & M & M & & 43 & \\
\hline Verbesina * & & & & & & & & & & $\mathrm{T}$ & $\mathrm{T}$ & & & & & & 29 & \\
\hline Gnaphalium * & & & & & & & & & & & & M & & $S$ & & & 29 & \\
\hline \multicolumn{19}{|l|}{ Aquifoliaceae } \\
\hline Ilex argentina* & & & & & & & & & & & $\mathrm{T}$ & & & $\mathrm{T}$ & $\mathrm{T}$ & & 43 & \\
\hline Brassicaceae & & M & & & & & & 14 & & & & & $\mathrm{~T}$ & & & & 14 & \\
\hline \multicolumn{19}{|l|}{ Buddlejaceae } \\
\hline Buddleja* & & & & & & & & & & & & & & & & & & $\mathrm{T}$ \\
\hline Cactaceae & & & & & & & & & & S & & & & & & & 14 & \\
\hline \multicolumn{19}{|l|}{ Caprifoliaceae } \\
\hline Tipo Sambucus* & & & & & & & & & & M & & & & & & & 14 & \\
\hline \multicolumn{19}{|l|}{ Eleocarpaceae } \\
\hline Crinodendron tucumanun* & $D$ & M & $\mathrm{T}$ & $\mathrm{T}$ & & & & 57 & $\mathrm{~T}$ & S & $\mathrm{T}$ & M & & & & & 43 & \\
\hline Euphorbiaceae & & & & & M & S & & 29 & & & & & & & & & & \\
\hline Sapium haematospermum* & & & $\mathrm{T}$ & & & & $\mathrm{T}$ & 29 & & & & & & & & & & \\
\hline Sebastiania* & & M & $\mathrm{T}$ & $\mathrm{T}$ & M & M & $\mathrm{T}$ & 86 & M & & M & $\mathrm{T}$ & M & $\mathrm{T}$ & $\mathrm{T}$ & M & 86 & $S$ \\
\hline \multicolumn{19}{|l|}{ Escalloniaceae } \\
\hline Escallonia schreiteri* & & & & & & & & & & M & & $\mathrm{T}$ & M & $\mathrm{T}$ & & & 57 & M \\
\hline Fabaceae & & & & & & & & & & & & M & & $\mathrm{T}$ & M & & 43 & \\
\hline Acacia aroma* & & & & & $\mathrm{T}$ & $\mathrm{T}$ & & 29 & $\mathrm{~T}$ & & & $\mathrm{~T}$ & & & & & 14 & $\mathrm{~T}$ \\
\hline Anadenanthera colubrina* & & & $\mathrm{T}$ & & $\mathrm{T}$ & $\mathrm{T}$ & $\mathrm{T}$ & 57 & $\mathrm{~T}$ & & & M & $\mathrm{T}$ & & & & 29 & \\
\hline Mimosa farinosa* & & & $\mathrm{T}$ & & & & & 14 & & & & & & & & & & \\
\hline Mimosa* & & & & & & M & M & 29 & & & & & & & M & & 14 & \\
\hline Parapiptadenia excelsa* & $\mathrm{T}$ & M & M & $\mathrm{T}$ & $S$ & M & M & 100 & $\mathrm{~T}$ & & $\mathrm{~T}$ & & & & & & 14 & $\mathrm{~T}$ \\
\hline \multicolumn{19}{|l|}{ Lamiaceae } \\
\hline Cantinoa* & & & & & & & M & 14 & & & & & & & & & & \\
\hline Leonurus japonicus* & & & & & & & & & & & & & $\mathrm{T}$ & & & & 14 & \\
\hline
\end{tabular}


Bol. Soc. Argent. Bot. 51 (3) 2016

\begin{tabular}{|c|c|c|c|c|c|c|c|c|c|c|c|c|c|c|c|c|c|c|}
\hline \multirow{3}{*}{ TIPOS POLINICOS } & \multicolumn{9}{|c|}{ 2011-2012 } & \multicolumn{9}{|c|}{$2012-2013$} \\
\hline & \multicolumn{8}{|c|}{ Ml } & \multirow{2}{*}{$\begin{array}{c}\text { MM } \\
\text { D }\end{array}$} & \multicolumn{8}{|c|}{ MI } & \multirow{2}{*}{$\begin{array}{c}\mathrm{MM} \\
\mathrm{N}\end{array}$} \\
\hline & S & 0 & $\mathrm{~N}$ & $\mathrm{D}$ & $E$ & $\mathrm{~F}$ & M & FO\% & & $S$ & 0 & $\mathrm{~N}$ & $\mathrm{D}$ & $E$ & $\mathrm{~F}$ & M & FO $\%$ & \\
\hline \multicolumn{19}{|l|}{ Malvaceae } \\
\hline Tipo Abutilon* & & & & & & & & & & & & & & & $\mathrm{T}$ & & 14 & \\
\hline Myrtaceae & & & & & & M & M & 29 & & & & & & & & & 29 & \\
\hline Blepharocalyx salicifolius* & $\mathrm{T}$ & M & D & D & S & $S$ & M & 100 & $\mathrm{D}$ & & & $S$ & $\mathrm{~T}$ & $\mathrm{~T}$ & & & 43 & \\
\hline Eucalyptus & & M & & & $S$ & M & $T$ & 57 & M & $\mathrm{T}$ & & & $\mathrm{T}$ & M & & M & 57 & \\
\hline \multicolumn{19}{|l|}{ Oleaceae } \\
\hline Ligustrum & & & & $\mathrm{T}$ & $\mathrm{T}$ & $\mathrm{T}$ & & 43 & & & & & & & $D$ & $D$ & 29 & \\
\hline \multicolumn{19}{|l|}{ Ranunculaceae } \\
\hline Clematis* & & & & & & & & & & & & & & & $T$ & M & 29 & \\
\hline Rhamnaceae & & & & & & & $S$ & 14 & & & & & $S$ & $\mathrm{~T}$ & & & 29 & \\
\hline Tipo Scutia- Condalia* & & & & & & & & & & $\mathrm{T}$ & D & & & & & & 29 & $S$ \\
\hline Rosaceae & & & & & & & & & & & & & $\mathrm{T}$ & & & & 14 & \\
\hline \multicolumn{19}{|l|}{ Rutaceae } \\
\hline Zanthoxylum* & $\mathrm{T}$ & & & & & & $S$ & 29 & $\mathrm{~T}$ & & & & M & & & & 14 & $\mathrm{~T}$ \\
\hline \multicolumn{19}{|l|}{ Salicaceae } \\
\hline Salix humboldtiana* & M & M & $T$ & M & $\mathrm{T}$ & $T$ & M & 100 & $\mathrm{~T}$ & M & $\mathrm{T}$ & $\mathrm{T}$ & M & $\mathrm{T}$ & $\mathrm{T}$ & & 86 & M \\
\hline Xilosma pubescens* & & $T$ & & & & & & 14 & & & & & & & & & & \\
\hline \multicolumn{19}{|l|}{ Sapindaceae } \\
\hline Allophylus edulis* & $\mathrm{T}$ & $S$ & $S$ & M & $\mathrm{T}$ & M & $S$ & 100 & M & & M & $\mathrm{T}$ & M & $\mathrm{T}$ & & & 57 & $S$ \\
\hline Serjania* & & & & & & & $T$ & 14 & & & & & & & & & & \\
\hline Solanaceae & & M & & & & & & 14 & & & & & $\mathrm{~T}$ & & & & 14 & \\
\hline Vassobia breviflora* & & & & & & & & & & & & M & M & & & & 29 & \\
\hline Verbenaceae & & & & & & & & & & & & & & & & & & $T$ \\
\hline \multicolumn{19}{|l|}{ Vitaceae } \\
\hline Tipo Cissus* & & & & & & & & & & & & $\mathrm{T}$ & M & $S$ & & & 43 & \\
\hline Liliopsida & & & & $\mathrm{T}$ & & & & 14 & & & & M & & & & & 14 & \\
\hline Indeterminados & $T$ & M & & $T$ & & & & 43 & & & & & & & & & & \\
\hline \multicolumn{19}{|l|}{ PLANTAS DE POLINIZACIÓN } \\
\hline \multicolumn{19}{|l|}{ ANEMÓFILA O SIN NÉCTAR } \\
\hline \multicolumn{19}{|l|}{ Celtidaceae } \\
\hline Celtis* & & + & & + & + & + & & & & & + & + & & & & & & + \\
\hline \multicolumn{19}{|l|}{ Juglandaceae } \\
\hline Juglans australis* & & & & & & & & & & + & + & + & + & & & & & + \\
\hline Moraceae & & & & & & & & & & & & & & & & & & \\
\hline Morus & + & & + & & & & & & & + & & & & & & & & + \\
\hline Oleaceae & & & & & & & & & & & & & & & & & & \\
\hline Fraxinus & & & & & & & & & & + & & & & & & & & \\
\hline Poaceae & & + & & & + & & & & & & & & & & & & & \\
\hline Zea mays & & & & & & & & & & & & & & & + & + & & \\
\hline Urticaceae & & & & & & & & & & & & + & & & & & & + \\
\hline RIQUEZA POLINICA & 9 & 15 & 13 & 13 & 13 & 14 & 16 & & 11 & 14 & 12 & 19 & 17 & 13 & 11 & 6 & & 17 \\
\hline
\end{tabular}




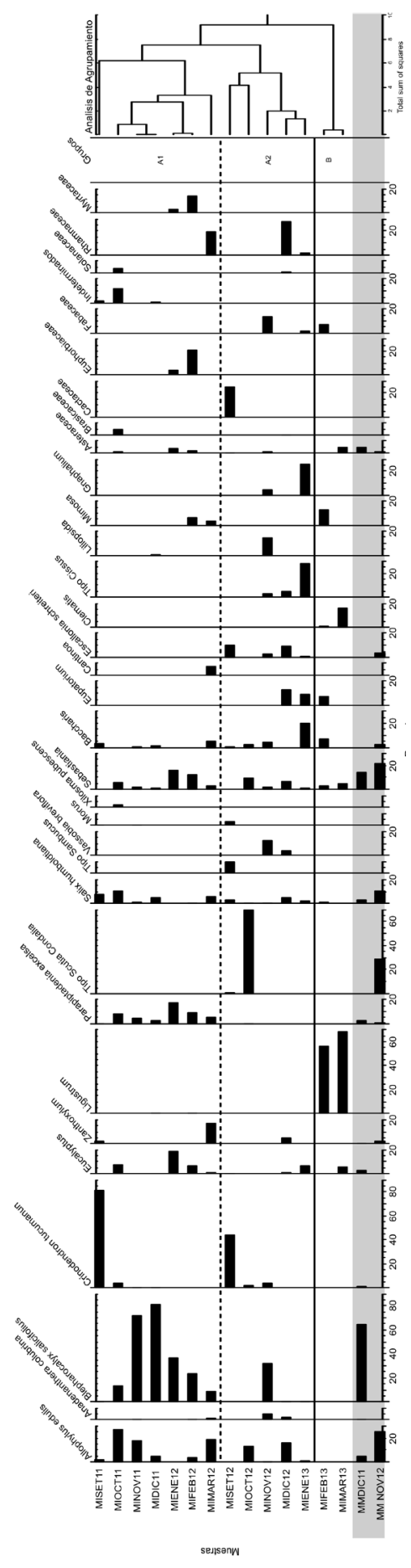

Fig. 1. Diagrama polínico y Análisis de agrupamiento de mieles inmaduras de Apis mellifera (períodos productivos Set 2011- Mar 2012 y Set 2012 Mar2013). Representación de los tipos polínicos con porcentajes superiores al $3 \%$.
Solanaceae y un tipo polínico indeterminado (en menor importancia). Para el periodo 20122013 se encontraron: Senecio, Eucalyptus, Verbesina e Ilex argentina en valores trazas, el tipo polínico Sambucus en menor importancia y Cactaceae como secundario. Un caso particular es la representatividad de Crinodendron tucumanum, que se encuentra con un valor de $42 \%$ en el mes de setiembre y ausente en la miel madura.

\section{Origen geográfico}

Los tipos polínicos que caracterizan a las muestras analizadas son Blepharocalyx salicifolius, Allophylus edulis, tipo Scutia Condalia, Crinodendron tucumanum, Parapiptadenia excelsa, Zanthoxylum, entre otros.

Respecto a los análisis cuantitativos, no existen diferencias significativas entre las concentraciones polínicas de los dos periodos estudiados.

\section{Discusión}

Principales fuentes de néctar para las colmenas

Las familias botánicas que presentaron mayor riqueza polínica en las mieles inmaduras fueron Asteraceae y Fabaceae. Estos resultados concuerdan con otros trabajos realizados en la provincia de Jujuy tanto para mieles inmaduras (Burgos \& Sánchez, 2014) como maduras (Sánchez \& Lupo, 2006, 2011; Sánchez, 2013).

En la familia Asteraceae, Baccharis se destacó como un recurso nectarífero importante para las colmenas, al igual que lo observado por Sánchez (2013) en las diferentes zonas apícolas de Jujuy. El género presenta especies usualmente de hábito herbáceo y arbustivo, cuyas floraciones son utilizadas como fuentes de néctar y polen; por ejemplo, en sectores de la provincia fitogeográfica chaqueña argentina, las especies presentan floraciones consecutivas que abarcan la primavera (B. dracunculifolia DC.), el verano y el otoño ( $B$. breviseta DC., B. trinervis Pers., B. salicifolia (Ruiz \&Pav.) Pers., B. punctulata DC. y B. notosergila Griseb.) (Cabrera et al., 2013, Vossler et. al., 2014). Por otra parte Cabrera (2006), Caccavari \& Fagúndez (2010), Costas et al., (2013) y Fagúndez (En prensa) mencionan el tipo Baccharis en mieles maduras de Apis mellifera en distintas clases de frecuencia. En áreas alrededor del delta del 
Bol. Soc. Argent. Bot. 51 (3) 2016

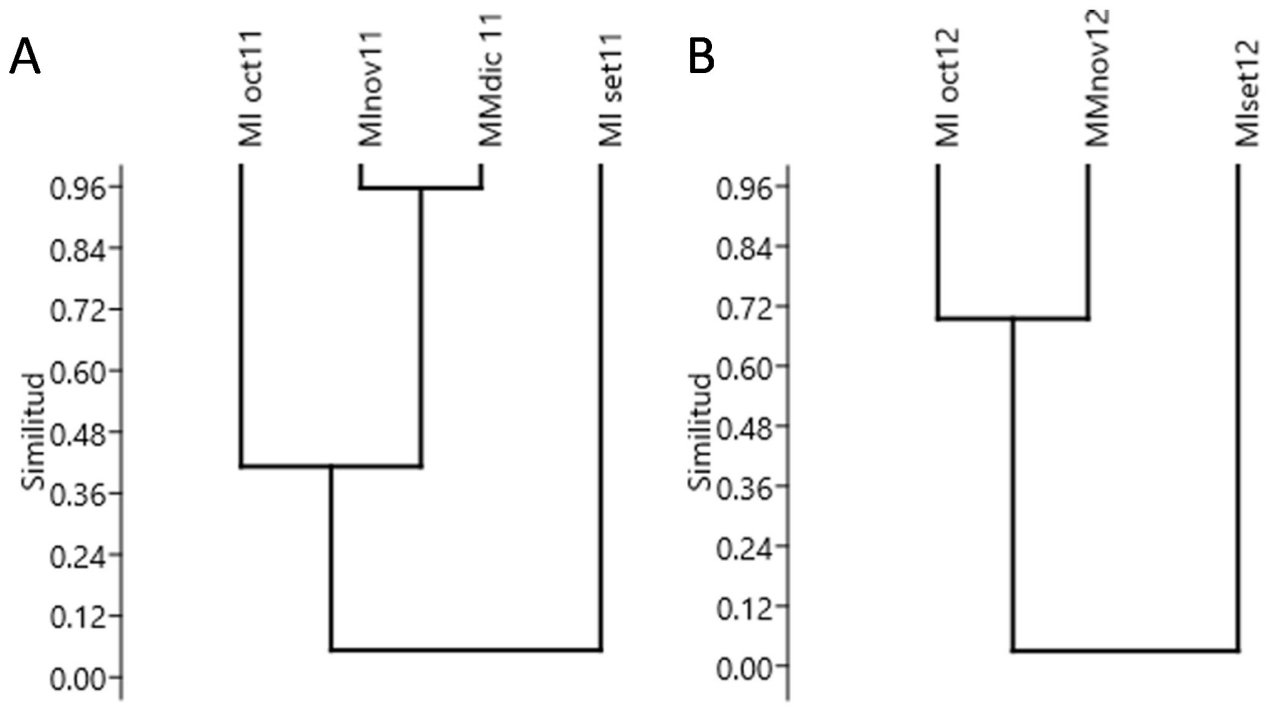

Fig. 2. Análisis de agrupamiento de las muestras de miel inmaduras precedentes a la muestra de miel madura (MI) y cosechada (MM) A. Periodo 2011-2012. B. Periodo $2012-2013$.

Paraná, en la provincia de Entre Ríos se producen mieles monoflorales de este taxón (Basilio \& Romero, 1996; Fagúndez \& Caccavari, 2006; Fagúndez et al., 2015). En el caso de las abejas "sin aguijon", Flores \& Sánchez (2010) identifican como dominante a este recurso en mieles de Tetragonisca angustula. Vossler (2015) y Dalmazzo \& Vossler (2015) también lo registran en mieles de otras especies de melipónidos como Tetragonisca fiebrigi y Augochlora amphitrite respectivamente, confirmando el valor melífero del género botánico. Respecto a Eupatorium, en este estudio ha sido identificado como polen de menor importancia, a diferencia de lo observado en estudios de mieles maduras de A. mellifera (Sánchez, 2013) y Plebeia intermedia Wille (Apidae, Meliponini) (Flores et al., 2015), donde es considerado un recurso nectarífero secundario mientras que en el Delta medio del Rio Paraná, Caccavari \& Fagúndez (2010) lo mencionan como un recurso dominante productor de mieles monoflorales. En mieles de la región Chaqueña, algunos autores destacan la importancia del tipo polínico Baccharis-Eupatorium para las colmenas de A. mellifera, logrando clasificar mieles inmaduras monoflorales (Salgado et al., 2014) o vincular su presencia con tipos polínicos dominantes, como ser Copernicia alba (palma), y con otros tipos polínicos (Cabrera et al., 2015).
Vossler et al. (2010) también lo menciona como un recurso visitado por Geotrigona argentina. En el caso de Gnaphalium, citado para la región como elemento traza en mieles de P. intermedia (Flores et al., 2015), en el área estudiada fue observado en la miel inmadura con clases de frecuencia de secundario a menor importancia, siendo ésta la primera referencia para mieles de A. mellifera.

En la familia Fabaceae, Parapiptadenia excelsa es considerado un tipo polínico relevante y de uso muy frecuente, formando parte de la asociación polínica indicadora de la zona apícola de Yungas de Jujuy (Sánchez, 2013). Para el Chaco Serrano es considerado un aporte nectarífero importante en mieles inmaduras de Apis mellifera (Burgos \& Sánchez, 2014) y su uso por otros ápidos fue mencionado por Flores \&Sánchez (2010) y Flores et al. (2015) en mieles maduras de Tetragonisca angustula y Plebeia intermedia en sectores yungueños de Salta. Otras Fabaceae observadas en este estudio fueron Anadentahera colubrina y Mimosa. En el primer caso, si bien está presente en bajas proporciones (polen de menor importancia y traza) y muy frecuente durante el periodo 2011-2012, se diferencia de lo observado en mieles de $A$. mellifera del Chaco Serrano jujeño (Burgos \& Sánchez, 2014) y en muestras de Tetragonisca angustula en las Yungas de Salta 


\section{V. Mendez et al. - Mieles inmaduras de las Yungas Oeste de Jujuy}

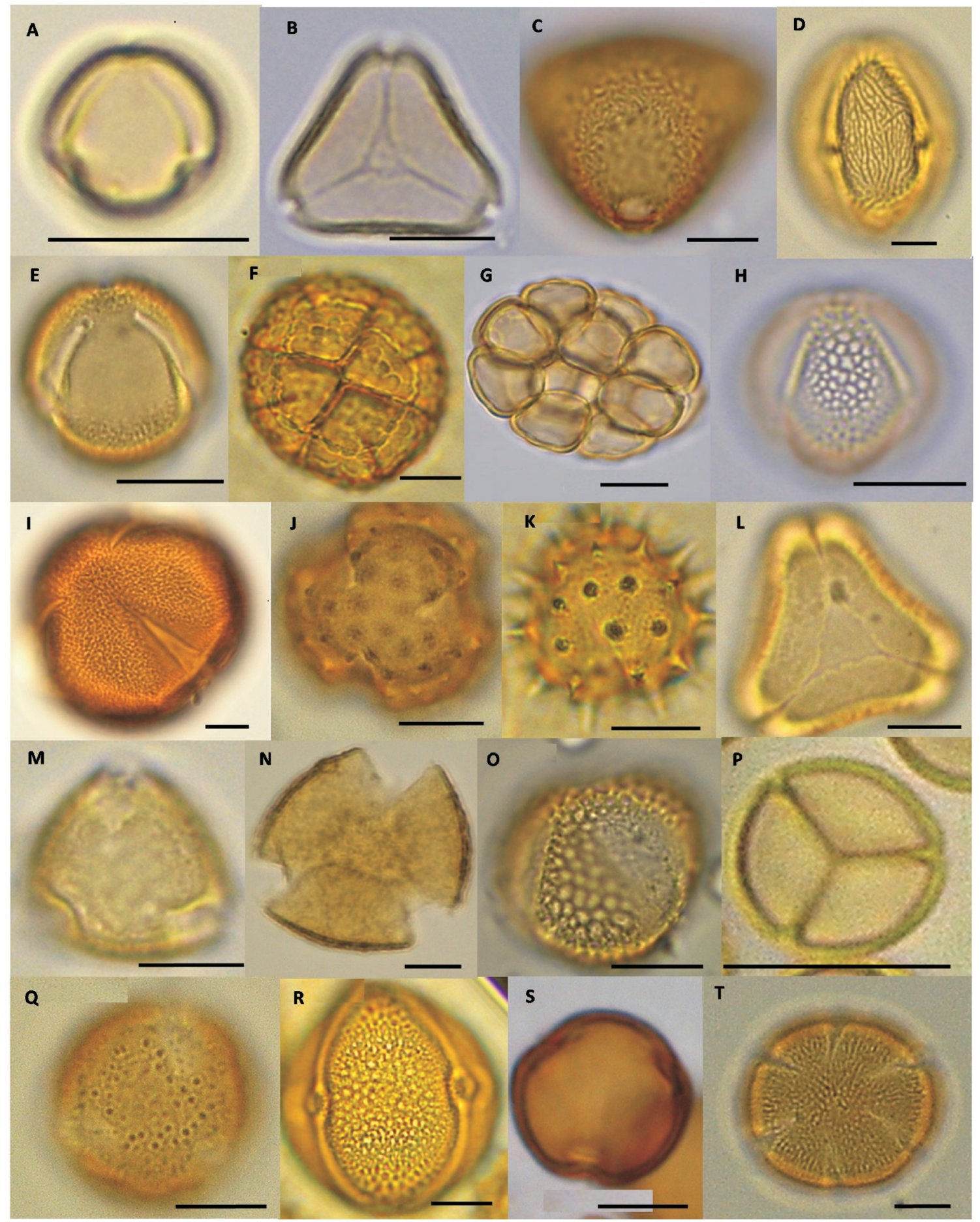

Fig. 3. Principales recursos nectaríferos utilizados por Apis mellifera L. en la localidad de Tilquiza. A: Crinodendron tucumanum. B: Blepharocalyx salicifolius. C: Allophylus edulis D: Zanthoxylum. E: Escallonia schreiteri. F: Anadenathera colubrina. G: Parapiptadenia excelsa. H: Salix humboldtiana. I: Sebastiania. J: Gnaphalium. K: Baccharis. L: Eucalyptus. M: Tipo Scutia/Condalia. N: Cactaceae. O: Tipo Sambucus. P: Mimosa. Q: Clematis. R: Tipo Cissus. S: Euphorbiaceae. T: Cantinoa. Escala A-Q: $10 \mu \mathrm{m}$. 
(Flores \& Sánchez, 2010) donde se encuentra como un taxón dominante. En el caso de Mimosa, presente en este estudio con valores de menor importancia, recientemente ha sido mencionado en mieles inmaduras y cargas corbiculares para el sector Este de las Yungas en Jujuy (Méndez et al., 2015). Su presencia en mieles maduras puede variar desde valores traza, en la región del Chaco (Salgado \& Pire, 1998), en el Delta medio del Rio Paraná (Caccavari \& Fagúndez, 2010) y en Diamante en Entre Ríos (Fagúndez, en prensa), a valores de menor importancia en el Espinal en Argentina (Fagúndez \& Caccavari (2006) y el Nordeste de Brasil (Freitas \& Silva, 2006). El género comprende especies con aptitud apícola (fuentes de néctar y polen), lo cual se observa en los trabajos de Sánchez (2013), Costas et al. (2013) y Burgos \& Sánchez (2014), donde se mencionan mieles monoflorales de M. debilis, Mimosa sp. y M. xanthocentra var. xanthocentra respectivamente.

De acuerdo a la dominancia de los tipos polínicos en las muestras, se destacaron las familias Rhamnaceae y Myrtaceae, las cuales tienen importancia melífera en otros sectores de Yungas y en el Chaco Serrano de la provincia de Jujuy (Sánchez \&Lupo, 2011; Sánchez, 2013). Las Rhamnaceae presentan interés en otras regiones fitogeográficas del país, como el Espinal (Andrada, 2003; Fagúndez \& Caccavari, 2006), el Monte en el valle de Río Negro (Tellería \& Forcone, 2000), en la Pampa (Naab y Tamame, 2007) y el Chaco (Salgado et al, 2014). En las mieles inmaduras analizadas se identificó como polen dominante al tipo Scutia/Condalia, que reúne a las especies Scutia buxifolia y Condalia buxifolia, ambas presentes en el área de estudio (Sánchez \& Lupo, 2011). En la provincia fitogeográfica Chaqueña, Salgado et al. (2014) identificaron a Scutia buxifolia como un importante recurso nectarífero para las colmenas, por su presencia dominante y secundaria en las mieles. Respecto a Myrtaceae, es considerada una de las principales familias proveedoras de néctar a "abejas sin aguijón" (Meliponini) de las Yungas en Salta (Flores \& Sánchez, 2010; Flores et al., 2015) y para otras zonas neotropicales (Ramalho et al., 1990). En este estudio, la dominancia de Blepharocalyx salicifolius en las mieles inmaduras de Apis mellifera coincide con el periodo de floración que presenta la especie (Legname \&Digilio, 1966) y con la abundancia del recurso en áreas cercanas al apiario, donde forma un mesobosque junto a Juglans australis entre los 1407 y 1453 msnm (Martin, 2014). La presencia de este recurso como polen dominante se observa en mieles inmaduras de A. mellifera de la localidad de El Fuerte (Dpto. Santa Barbara, Jujuy) (Méndez et al., 2015) y en mieles maduras de Plebeia intermedia en la localidad de Baritú (Dpto. Santa Victoria, Salta) (Flores et al., 2015). En el caso de Eleocarpaceae se menciona por primera vez la importancia de la familia como recurso melífero para el área de estudio, lo que no fue observado en estudios precedentes (Sánchez, 2013). Un tipo polínico significativo en las mieles inmaduras fue Crinodendron tucumanum, coincidente a lo observado en mieles maduras de sectores de Yungas septentrionales de Argentina (Flores, 2008) y en menor proporción en mieles de $P$. intermedia (Flores et al., 2015).

Como polen secundario, en orden decreciente de importancia se registraron Allophylus edulis, Zanthoxylum, tipo Cissus y Eucalyptus. Los dos primeros son considerados indicadores de la asociación polínica de la zona apícola de Yungas (Sánchez, 2013). En el caso de Allophylus edulis, muy frecuente, utilizado durante las temporadas estudiadas, constituye junto a Blepharocalyx salicifolius las principales fuentes de néctar de las colmenas de Plebeia intermedia en los bosques del norte de Salta (Flores et al., 2015). A su vez, junto a otros tipos polínicos (tipo Lithraea molleoides, Blepharocalyx salicifolius, Gleditsia amorphoides, Salix humboldtiana, Mimosa, Brassicaceae y Poaceae) son los principales recursos alimenticios para Apis mellifera, observado en mieles inmaduras y cargas corbiculares en el sector este de las Yungas de Jujuy (Méndez et al., 2015).

Respecto a Zanthoxylum, ha sido identificado en mieles de A. mellifera como polen dominante por Costas et al. (2013), de menor importancia por Sánchez \& Lupo, (2011) y con valores traza en mieles de Plebeia intermedia por Flores et al. (2015). El tipo Cissus se cita por primera vez en mieles de A. mellifera para la región del NOA, ya que en mieles de la zona central de Entre Ríos y el Delta Medio del Rio Paraná es citado como polen traza y de menor importancia (Fagúndez \& Caccavari, 2006; Caccavari \& Fagúndez, 2010) y en mieles de P. intermedia de las Yungas de Salta, Flores et al. (2015) lo mencionan como polen traza. 
Finalmente la presencia de Eucalyptus en la miel está relacionada con las plantaciones forestales en la zona (Braun Wilke et al., 2013) que contribuyen en la elaboración de las mieles. Sus especies son consideradas buenas fuentes de néctar para las abejas, permitiendo la obtención de mieles monoflorales tanto en la provincia de Jujuy (Sánchez, 2013), como en el resto del país (Forcone, 2008; Fagundez \& Caccavari, 2006; Cabrera, 2006; Forcone \& Tellería, 2000; entre otros). Además, estudios sobre origen botánico en cargas corbiculares, lo registraron como una importante fuente de polen en la provincia de Jujuy (Burgos et al., 2015; Méndez, 2014). En el sur de Brasil, se observó también su importancia polinífera tanto en colmenas de $A$. mellifera como en especies de meliponas (Luz et al., 2011; Hilgert Moreira et al., 2014).

Respecto al origen geográfico, los tipos polínicos que caracterizan a las muestras analizadas son Allophylus edulis, Baccharis, Blepharocalyx salicifolius, Parapiptadenia excelsa, Salix humboldtiana, Scutia/Condalia y Zanthoxylum, entre otros, todos ellos correspondientes a especies nativas de la zona y que integran la asociación polínica del sector Yungas Oeste (Sánchez, 2013).

El uso de néctar de especies nativas, también se observa en las mieles inmaduras de Apis mellifera en diferentes zonas del país (Naab \& Tamame, 2007; Salgado et al., 2014; Burgos \& Sánchez 2014), a diferencia de lo registrado en mieles del Delta inferior del Paraná (Basilio \& Romero, 2002) y el valle inferior del Río Chubut (Forcone et al., 2003), donde existe mayor uso de especies de origen exótico.

\section{Dinámica del ingreso de néctar a las colmenas}

En el análisis de los recursos nectaríferos a lo largo de las temporadas estudiadas, se determinó que durante el inicio de la primavera de 2011 los principales aportes de néctar para las colmenas provinieron de Crinodendron tucumanum y Allophylus edulis acompañados de Blepharocalyx salicifolius, Parapiptadenia excelsa, Salix humboldtiana y Sebastiania. A mediados y fines de la primavera (noviembre y diciembre), Blepharocalyx salicifolius fue el principal recurso de la colmena. Durante el inicio y mediados del verano (2011-2012), este tipo polínico se mantuvo presente en las muestras, lo cual se podría atribuir a una contaminación desde las celdillas de almacenamiento de polen de meses anteriores, debido a que la especie no se encuentra en floración durante esta época. Euphorbiaceae, Sebastiania, Parapiptadenia excelsa y Eucalyptus contribuyeron también a la colmena en este periodo. A fines de la temporada apícola, los principales ingresos de recursos fueron Allophylus edulis, Zanthoxylum y un tipo polínico correspondiente a la familia Rhamnaceae acompañados por Baccharis, Cantinoa, Mimosa, Parapiptadenia excelsa y Salix humboldtiana. Se observa que Allophylus edulis y Zanthoxylum no se encuentran en floración durante estos meses por lo que su presencia estaría relacionada a la contaminación en la colmena al igual que sucede en los meses anteriores con otros tipos polínicos.

En la primavera de 2012, al igual que la temporada anterior, Crinodendron tucumanum también fué el principal recurso en los inicios de esta estación, adquirendo relevancia el tipo Scutia/Condalia en el mes de octubre. A fines de la primavera Blepharocalyx salicifolius continuó siendo el principal ingreso (aunque en esta temporada apareció en menor proporción que en la temporada anterior) junto al tipo polínico Rhamnaceae. Otros recursos visitados fueron: Anadenanthera colubrina, Escallonia schreiteri, Vassobia breviflora Salix humboldtiana, Baccharis, Eupatorium, Gnaphalium, Sebastiania, Zanthoxylum, tipo Cissus, Liliopsida y Fabaceae.

En el inicio del verano los principales recursos fueron Baccharis, Gnaphalium y tipo Cissus. Una situación particular se observa en las muestras de mediados y fines del verano 2013 (con valores de concentración polínica bajos, entre 1700 y 3500 granos de polen $/ 10 \mathrm{~g}$ de miel), donde el principal tipo polínico identificado corresponde a Ligustrum. Si bien el periodo de floración de las especies de este género se menciona para los meses octubre, noviembre y diciembre (García, 2010; Romeo \& Entrocassi, 2012), en esta zona la floración persiste hasta fines de verano (Méndez et al., obs. pers.).

Por otra parte, la diferencia en la representatividad de los tipos polínicos identificados en las muestras de miel inmadura respecto a la miel madura correspondería al consumo que realiza la colmena para su crecimiento y desarrollo, como también se observa en apiarios de la provincia fitogeográfica Chaqueña (Salgado et al. 2014). 


\section{Conclusiones}

El trabajo realizado constituye el primer aporte al conocimiento del contenido polínico en mieles inmaduras de la primavera y verano de dos temporadas apícolas, para el sector oeste de las Yungas de Jujuy.

En el espectro polínico se observó la influencia de la flora nativa perteneciente al distrito de la Selva Montana de las Yungas durante los ciclos productivos estudiados, lo que posibilitaría la obtención de mieles uniflorales características del sector.

Las diferencias observadas entre el recurso utilizado por las abejas en los meses previos a la cosecha y el contenido polínico en las muestras comerciales, evidencian la importancia de este tipo de estudios, que permite determinar el recurso consumido en la colmena.

El conocimiento generado, podrá ser utilizado por los apicultores del sector, permitiendo comprender la dinámica del ingreso de néctar a las colmenas y valorar el recurso ofrecido por el bosque.

\section{Agradecimientos}

Los autores agradecen a los Proyectos SECTER UNJu: "Determinación de las zonas apícolas en la provincia de Jujuy mediante su caracterización botánica" y "Caracterización botánica y geográfica de las mieles y recursos polínicos de abejas melíferas nativas e introducidas en Yungas y Chaco de las provincias de Jujuy y Salta"; al CIN (Consejo Interuniversitario Nacional), al CONICET, al apicultor Ezequiel Verrastro y a los diferentes colaboradores en las tareas de campo.

\section{Bibliografia}

ANDRADA, A. C. 2003. Flora utilizada por Apis mellifera L. en el sur del Caldenal (Provincia Fitogeográfica del Espinal), Argentina. Rev. Mus. Argentino Cienc. Nat., n.s. 5: 329-336.

BARTH, O. M. 1989. O pólen no mel brasileiro. Ed. Luxor, Río de Janeiro, Brasil.

BASILIO, A. M. \& E. J. ROMERO. 1996. Contenido polínico en las mieles de la región del Delta del Paraná (Argentina). Darwiniana. 34: 113-120.

BASILIO, A. M. \& E. J. ROMERO. 2002. Variaciones anuales y estacionales en el contenido polínico de la miel de un colmenar. RIA. 31: 41 - 58.

BRAUN WILKE, R., E. E. SANTOS, L. P. PICCHETTI, M. T. LARRAN, G. F. GUZMAN, C. R. COLARICH \& C. A. CASOLI. 2013. Carta de aptitud ambiental de la provincia de Jujuy. Ediunju. San Salvador de Jujuy.

BURGOS M. G. \& A. C. SANCHEZ. 2014. Preferencias alimenticias en las mieles inmaduras de Apis mellifera en el Chaco Serrano (Jujuy, Argentina). Bol. Soc. Argent. Bot. 49: 41-50.

BURGOS M. G., SANCHEZ A. C. \& L. C. LUPO. 2015. Análisis polínico de cargas corbiculares de Apis mellifera del Chaco Serrano, Jujuy (Argentina). Lilloa. 52: 3-11.

CABRERA, A. L. 1976. Regiones Fitogeografías Argentinas. Enciclopedia Argentina de Agricultura y Jardinería. Ed. Acme. Buenos Aires.

CABRERA M.M. 2006. Caracterización polínica de las mieles de la provincia de Formosa, Argentina. Rev. Mus. Argentino Cienc. Nat., n. s. 8: 135 - 142

CABRERA, M., ANDRADA, A. \& L. GALLEZ. 2013. Floración de especies con potencial apícola en el Bosque Nativo Formoseño, Distrito Chaqueño Oriental (Argentina). Bol. Soc. Argent. Bot. 48: 477-491.

CABRERA, M., ANDRADA, A., GALlEZ, L. \& G. BALBARREY. 2015. Mieles de Palmares del Chaco Húmedo de Formosa, Argentina. Bol. Soc. Argent. Bot. (Supl.): 240.

CACCAVARI M. \& G. FAGUNDEZ. 2010. Pollen spectra of honeys from the Middle Delta of the Paraná River (Argentina) and their environmental relationship. Spanish Journal Agricultural Research. 8: 42-52.

COSTAS M. C., VERGARA-ROIG V. A. \& S. C. KIVATINITZ. 2013. A Melissopalynological study of artisanal honey produced in Catamarca (Argentina). Grana. 52: 229-237.

CHAFATINOS, T. \& A. NADIR. 1990. Los Suelos del NOA (Salta y Jujuy). Administración del Fondo Especial del Tabaco.

CHAMORRO GARCIA F. J., LEON BONILLA D. \& NATES PARRA G. 2013. El polen apícola como producto forestal no renovable en la Cordillera Oriental de Colombia. Colombia Forestal. 16: 5366.

DALMAZZO M. \& F. G. VOSSLER. 2015. Pollen host selection by a broadly polylectic halictid bee in relation to resource availability. Arthropod-Plant Interactions, 9: 253-262.

DE KLERK, P. \& H. JOOSTEN. 2007. The difference between pollen types and plant taxa: a plea for clarity and scientific freedom. Eiszeitalter und Gegenwart / Quaternary Science Journal. 56: 162-171. 
ERDTMAN, G. 1960. The acetolysis method, a revised description. Svensk. Bot. Tidskr. 54: $561-564$.

FAGUNDEZ, G. A. En prensa. Botanical and geographical characterization of honeys in Diamante, Entre Rios, Argentina. Palynology.

FAGUNDEZ, G. A. \& M. A. CACCAVARI. 2006. Pollen analysis of honeys from the central zone of the Argentine province of Entre Rios. Grana 45: 305-320.

FAGUNDEZ G., GURINI L., LOPEZ V. C., BERTOS M., TRUJILLO C., BARBERO P. \& D. BETLLER. 2015. Caracterización botánica y geográfica de mieles del Delta medio e inferior del Rio Paraná (Argentina).Libro de Resumenes XVI Simposio Argentino de Paleobotanica y Palinologia, La Plata 2015.

FELLER DEMALSY, M., J. PARENT \& A. STRACHAN. 1987. Microscopic analysis of honeys from Alberta, Canada. J. Apic. Res. 26: 123-132.

FLORES F. F., 2008. Informe Técnico: Tipificación de mieles de Tetragonica angustula. Localidad Los Naranjos. Oran. Salta. Fundación ProYungas.

FLORES, F. F. \& A. C. SÁNCHEZ. 2010. Primeros resultados de caracterización botánica de mieles de Tetragonisca angustula Latreille (Apidae, Meliponinae) criadas en la localidad Los Naranjos Orán - Salta. Bol. Soc. Argent. Bot. 45: 81-91.

FLORES F. F., LUPO L. C. \& N. I. HILGERTH. 2015. Recursos tróficos utilizados por Plebeia intermedia (Apiade, Meliponini) en la localidad de Baritú, Salta, Argentina. Caracterización botánica de sus mieles. Bol. Soc. Argent. Bot. 50: 515-529.

FORCONE, A. 2008. Pollen analisys of honey from Chubut (Argentinean Patagonia). Grana 47: $147-$ 158.

FORCONE A., O. BRAVO. \& M. G. AYESTARAN. 2003. Interaannual variation in the pollinic spectrum of honey from the lower valley of the River Chubut (Patagonia, Argentina). Spanish Journal of Agricultural Research. 1: 29-36.

FREITAS B. M. \& E.M. S. SILVA. 2006. Potencial apícola da vegetação do semi - árido brasileiro. En: SANTOS, F. A. R. (ed.). Apium Plantae, pp 19-32. IMSEAR, Recife.

GARCIA M. E. 2010. Aeropalinologia de la ciudad de Yerba Buena, provincia de Tucumán. (Argentina). Acta Botánica Malacitana. 35: 115-131.

GRIMM, E.C. 2011. Tilia sotware. Version 1.7.16. Illinois State Museum. Research \& and Collection Center. Springfield, Illinois.

HAMMER, Ø., HARPER, D. A. T. \& P. D. RYAN. 2001. PAST: Paleontological Statistics Software Package for education and data analysis. Palaeontologia Electronica 4: 9 pp. Diponible en: http://folk.uio.no/ ohammer/past/.
HEUSSER, C. J. 1971. Pollen and Spores of Chile. Tucson. Arizona.

HILGERT MOREIRA S. B., NASCHER C. A., CALLEGARI JACQUES S. M. \& B. BLOCHTEIN. 2014. Pollen resources and trophic niche breadth of Apis mellifera and Melipona obscurior (Hymenoptera, Apidae) in a subtropical climate in the Atlantic rain forest of southern Brazil. Apidologie 45: 129-141.

JOOSTEN, H. \& P. DE KLERK. 2002. What's in a name? Some thoughts on pollen classification, identification, and nomenclature in Quaternary palynology. Review of Palaeobotany and Palynology 122: 29-45.

LEGNAME, P. \& A. DIGILIO.1966. Los Árboles indígenas de la Provincia de Tucumán. Opera Lilloana. 15: 1-283.

LOUVEAUX, J.A. MAURIZIO \& G. VORWHOL. 1978. Methods of Melissopalynology by International Commission of Bee Botany of IUBS. Bee World 59: 139-157.

LUZ, C.F.P., FERNANDES-SALOMÃO, T. M., LAGE, L. G. A., RESENDE, H. C., TAVARES, M. G. \& L. A. O. CAMPOS. 2011. Pollen sources for Melipona capixaba Moure and Camargo: an endangered Brazilian stingless bee. Psyche: A Journal of Entomology. 2011, 1-7

MAGYP. Ministerio de Agricultura, Ganadería y Pesca de la Nación. Disponible en (http://www. minagri.gob.ar/site/desarrollo_rural/producciones regionales/00_origen_animal/00_apicultura/_items/ estadisticas.htm)(marzo 2016)

MARKGRAF, V. \& H. D’ANTONI. 1978. Pollen Flora of Argentina. The University of Arizona Press. Arizona, EE.UU.

MARTIN C. M. 2014. Estudio de la vegetación a lo largo de un gradiente altitudinal en el Bosque Pluviestacional de Chijra (Jujuy, Argentina). Tesina de Grado. Universidad Nacional de Jujuy.

MENDEZ, M. V. 2014. Recurso nectaríferos y poliníferos utilizados por Apis mellifera L. en la localidad de Tilquiza, Jujuy, Argentina. Tesina de Grado. Facultad de Ciencias Agrarias. Universidad Nacional de Jujuy.

MÉNDEZ, M. V., SÁNCHEZ, A. C. \& L. C. LUPO. 2015. Avances en la caracterización palinológica de mieles inmaduras y cargas corbiculares en el

sector este de las yungas de Jujuy (Argentina). Bol. Soc Argent. Bot. (Supl.): 244.

MOAR, N. T., 1985. Pollen Analysis of New Zealand Honey. N. Z. Journ. Agric. Res. 28: 39-70.

MORENO, C. E. 2001. Métodos para medir la biodiversidad. M\&T-Manuales y Tesis SEA, Zaragoza.

NAAB, O. \& M. A. TAMAME. 2007. Flora apícola 
primaveral en la región del Monte de la provincia de la Pampa (Argentina). Bol. Soc. Argent. Bot. 42: 251-259.

PIRE, S. M., L. M. ANZÓTEGUI \& G. A. CUADRADO. 1998. Flora Polínica del Nordeste Argentino 1. EUDENE-UNNE. Corrientes.

PIRE, S. M., L. M. ANZÓTEGUI \& G. A.CUADRADO. 2002. Flora Polínica del Nordeste Argentino 2. EUDENE-UNNE. Corrientes.

PIRE, S. M., L. M. ANZÓTEGUI \& G. A.CUADRADO. 2006. Flora Polínica del Nordeste Argentino 3. EUDENE-UNNE. Corrientes.

RAMALHO, M., KLEINERT GIOVANNINI, A. \& V. L. IMPERATRIZ FONSECA. 1990. Important bee plants for stingless bees (Melipona and Trigonini) and Africanized honeybees (Apis mellifera) in neotropical habitats: a review. Apidologie 21: 469488.

ROMEO A. R. \& ENTROCASSI. 2012. Los arboles de la ciudad de San Salvador de Jujuy, Provincia de Jujuy, Argentina (Primera Parte). EDIUNJU. Jujuy. Argentina.

SALGADO C. R. \& S. M. PIRE 1998. Análisis polínico de las mieles del noroeste de la provincia de Corrientes (Argentina). Darwiniana. 36: 87-93

SALGADO C. R., G. PIESKO \& M. C. TELLERIA. 2014. Aporte de la melisopalinología al conocimiento de la flora melífera de un sector de la Provincia Fitogeográfica Chaqueña. Bol. Soc. Argent. Bot. 49: 513-524.

SANCHEZ, A. C. 2013. Caracterización Botánica y Geográfica de las mieles de Apis mellifera L. en la Provincia de Jujuy. Tesis Doctoral. Facultad de Ciencias Naturales. Universidad Nacional de Salta.

SÁNCHEZ, A. C. y L. C. LUPO. 2006. Tipos polínicos presentes en mieles uniflorales de la provincia de Jujuy, Argentina. 1er. Congreso Argentino de Apicultura: 53.
SÁNCHEZ, A. C. \& L. C. LUPO.2011. Origen botánico y geográfico de las mieles de El Fuerte Departamento de. Santa Bárbara, Jujuy, Argentina. Bol. Soc. Argent. Bot. 46:105-111.

STOCKMARR, J. 1971. Tablets with spores used in absolute pollen analysis. Pollen et spores 13: 615 $-621$.

TELLERIA, M. C. \& A. FORCONE. 2000. El polen de las mieles del Valle de Rio Negro, provincial fitogeográfica del Monte. Argentina. Darwiniana 38: 273-277.

VOSSLER F. G. 2015. Small pollen grain volumes and sizes dominate the diet composition of three south american subtropical stingless bees. Grana. 54: 68-81.

VOSSlER F. G., TELlERIA M. C. \& M. CUNNINGHAM. 2010. Floral resources foraged by Geotrigona argentina (Apidae, Meliponini) in the Argentine Dry Chaco forest. Grana. 49: 142-453.

VOSSLER F. G., FAGUNDEZ G. A. \& D. C. BLETTLER. 2014. Variability of Food stores of Tetragonisca fiebrigi (Schwarz) (Hymenoptera: Apidae: Meliponini) from the Argentine Chaco base don pollen analysis. Sociobiology. 61: 449-460

ZULOAGA, F. O., O. N. MORRONE, M. J. BELGGRANO, C. MARTICORENA \& E. MARCHESI. (eds). 2008. Catálogo de las PlantasVasculares del Cono Sur (Argentina, Sur de Brasil, Chile, Paraguay y Uruguay).Monogr. Syst. Bot. Missouri Bot. Gard. 107: I-XCVI, 1-983; 107: I-XX, 985-2286; 107: I-XXI, 2287-3348. http:// www2.darwin.edu.ar/Proyectos/FloraArgentina/ FA.asp (Cons. 23/04/2016).

Recibido el 30 de diciembre de 2015, aceptado el 25 de mayo de 2016. 